\title{
Research on the application of Chinese traditional pattern cloud pattern in Landscape Sketch
}

\author{
Zhigang $\mathrm{Li}^{1,}$ a, Zhonggui Yang ${ }^{1, \mathrm{~b}^{*}}$ \\ ${ }^{1}$ Wuhan University of Technology, Wuhan, China
}

\begin{abstract}
This paper discusses the application of traditional auspicious pattern cloud pattern in landscape sketch design. On the one hand, the purpose is to inherit the form aesthetic feeling and cultural connotation of cloud pattern, so as to apply the traditional cultural innovation in today's design and show our cultural confidence; on the other hand, to provide new ideas for the form innovation of landscape sketch, so as to design a set of cultural It is of great significance to integrate the value and aesthetic conception of landscape sketch, so as to meet the requirements of today's people for high-quality life. This electronic document is a "live" template. The various components of your paper [title, text, heads, etc.] are already defined on the style sheet, as illustrated by the portions given in this document.
\end{abstract}

\section{ReLATED CONCEPTS}

\subsection{The connotation of traditional moire}

Moire is a kind of expression of cloud in form. From the perspective of modern dialectical science, cloud is a physical phenomenon in nature, which is formed by the accumulation of water vapor. It can directly affect the growth of crops, as well as people's production and life. It's obvious that people's livelihood depends on simple farming and underdeveloped social conditions. Therefore, people have a sense of awe for the cloud. They deem that the cloud implies the natural law and controls the survival and development of all things in the world. Therefore, the cloud gradually. Gradually, the cloud deduces the traditional auspicious concept beyond human perception, and the cloud pattern is used as a kind of material in some clothing, architecture and various crafts.

Traditional moire is not the same in modeling. On the view of art design, moire pattern has the remarkable features of curl shape and flowing band combination. Owing to the arc itself has the form meaning and suggestibility of expansion, extension and circulation, plus the density change of line transformation, the rapid structural change and the sense of rhythm, the moire pattern can also give people a flexible feeling. Moreover, because of the expansion, extension and circulation of the arc itself, as well as the density change of the line transformation, the rapid structural change and the sense of rhythm, the moire pattern can also give people a kind of flexible sensation, so it can make people feel a kind of gorgeous transcendence.

\subsection{Basic concept of landscape sketch}

Landscape sketch is a punchline in environmental space. It is most commonly used in landscape design, environmental art design, landscape architecture design and other disciplines and industries. It generally refers to small buildings, urban sculpture, outdoor sculpture in outdoor landscape, including flower pools, tree pools, landscape walls, sitting boards, lamps, signs and other small structures or devices. Landscape sketch is not only closely related to our life, work and study, but also reflects the development of culture, economy, technology, science, technology and aesthetics of urban environment. Landscape sketch not only has practical use function value, but also contains aesthetic artistic value.

\subsection{The relationship between traditional cloud pattern and landscape sketch}

After thousands of years of continuous development, moire has a different fusion in each historical period, so it has different characteristics and cultural attributes in each specific historical period. Accordingly, the form and cultural implication of Moire has been constantly enriched and developed, so today, the traditional moire still exudes infinite artistic charm. Therefore, the cloud pattern as a design element of landscape sketch, On the one hand, as a design element of landscape sketch, the cloud pattern can bring a valuable source of inspiration to the design and creation of landscape sketch, designing an original form of landscape sketch. At the same time, it can inject more cultural connotation into the landscape sketches and give endow more auspicious meanings, thus causing cultural resonance. On the other hand, the research and redesign of cloud pattern can not only

\footnotetext{
ae-mail: 188796263@qq.com
}

*be-mail: 13983222754@qq.com 
explore the profound connotation of cloud pattern, but also explore its evolution. It is also a kind of transmission of traditional culture and a concrete expression of cultural confidence.

\section{EVOLUTION AND CHARACTERISTICS OF TRADITIONAL MOIRE}

\subsection{Primitive period}

People have different understandings of the traditional pattern of moire, and their understandings have improved with the progress of cultural level and the times. most studies on the origin of Moire believe that it is the imitation of nature by human beings, the objective reflection of natural phenomena by human beings, and at the same time, it is integrated into the subjective imagination of human beings; some people think that moire is produced in labor; while some people believe that it is the result of human activities. It is believed that the origin of moire is a human instinct or an expression form of primitive religious belief and primitive concept. There are different opinions, but there seems to be some truth. However, there has not been a unified point of view, which can only prove that the emergence and evolution of moire is closely related to human aesthetic consciousness.

From the perspective of archeology, the painted pottery has been used as a kind of primitive decoration in the primitive society. At this time, the pattern expression is mainly wave pattern, vortex pattern and concentric circle, which has the characteristics of simple, rough and vivid. Although the pattern at this time is very close to today's recognized cloud pattern in forms and formats, there is no direct text to confirm the consistency between the two. However, from the external aesthetic form and wheel frame of the pattern, we can see a possibility of the origin of cloud pattern, and at the same time, it can also show the ancient people's understanding and understanding of nature.

\subsection{Shang and Zhou Dynasties}

Shang-Zhou dynasty is an important historical period of cloud pattern development. Because the prevailing cloud and thunder pattern in this historical period was considered as the early form of cloud pattern by experts and scholars. At this time, the basic form of cloud and thunder pattern is similar to the original spiral pattern. It is composed of convoluted lines as the backbone, which has the characteristics of coarseness and toughness. Its specific manifestations include single spiral pattern, counter spiral pattern and S-shaped pattern and it is generally used in bronzes. On the one hand, it is similar to the original spiral pattern in its structure, thus showing a certain internal relationship; on the other hand, it has begun to be used as a shading or auxiliary pattern, thus beginning to have a clear primary and secondary relationship.

\subsection{Chu and Han Dynasties}

Cloud pattern developed into Chu Han period, and added "cloud tail", which has new features in form. The increase of this feature makes cloud pattern show certain speed and strength. The specific forms of cloud pattern in this period include "cloud Qi pattern" and banded cloud pattern, which has the characteristics of freedom, arbitrariness and romance. The appearance of "cloud tail" also coordinated with the immortal thought of the society at that time. If the cloud pattern with the tail of cloud is combined with the immortal and other animals, it can achieve the effect of light and slim and flying clouds. In addition, the Royal aristocracy showed a love for cloud patterns, which was embodied in the decoration of the palace with a large number of cloud patterns, so it indirectly promoted the inheritance of cloud patterns.

\subsection{Tang and Song Dynasties}

The most representative pattern at this time is the flower pattern, which has the characteristics of mellow cloud head and elegant cloud tail. The appearance of Moire also laid a foundation for the composition of composite moire. In the Song Dynasty, the general form of cloud pattern still maintained the style of Tang Dynasty cloud pattern. Based on this, some cloud heads and cloud hooks with changing curves and circles were added to the details to enrich the style of clouds. Influenced by the unique artistic trend of the Song Dynasty, there are some natural and loose modeling styles of cloud pattern in the Han Dynasty, which are lively, concise and flexible, and have the beauty of plastic arts.

\subsection{Ming and Qing Dynasties}

In the Ming and Qing Dynasties, there was not much change in the shape of moire. The most significant styles in this period were Tuan moire and stacked moire, and their main characteristics were multi-layer superposition, focusing on the sense of hierarchy, symmetry and regularity. The appearance of folding cloud pattern is also closely related to the pursuit of exquisite craftsmanship and the complicated and rich decoration in Qing Dynasty. In addition, the cloud pattern of this period was mostly used for wealth, longevity and other subjects, showing people's respect for its auspicious meaning.

TABLE 1. Analysis on the historical evolution of Moire

\begin{tabular}{|c|c|c|c|}
\hline \multicolumn{3}{|c|}{ Analysis on the historical evolution of Moire } \\
\hline period & Style moire & characteristics & sample \\
\hline $\begin{array}{c}\text { Primitive } \\
\text { period }\end{array}$ & $\begin{array}{c}\text { Motif rotatif } \\
\text { original }\end{array}$ & $\begin{array}{c}\text { pure } \\
\text { vivid }\end{array}$ \\
\hline $\begin{array}{c}\text { Shang and } \\
\text { Zhou } \\
\text { Dynasties }\end{array}$ & S-shaped pattern & $\begin{array}{c}\text { concise } \\
\text { robust and strong }\end{array}$ \\
\hline $\begin{array}{c}\text { Chu and } \\
\text { Han } \\
\text { Dynasties }\end{array}$ & Cloud pattern & comfortable and clear \\
\hline $\begin{array}{c}\text { Tang and } \\
\text { Song } \\
\text { Dynasties }\end{array}$ & Double check & $\begin{array}{c}\text { liberty } \\
\text { romantic } \\
\text { sense of order }\end{array}$ \\
\hline $\begin{array}{c}\text { Ming and } \\
\text { Qing } \\
\text { Dynasties }\end{array}$ & $\begin{array}{c}\text { Superimposed } \\
\text { cloud pattern }\end{array}$ & $\begin{array}{c}\text { sense of weight } \\
\text { sense of streaming } \\
\text { sense of streaming }\end{array}$ \\
\hline
\end{tabular}




\section{THE APPLICATION VALUE OF TRADITIONAL CLOUD PATTERN IN LANDSCAPE PRODUCTS}

\subsection{Innovation of incentive form}

From the initial spiral pattern to the group cloud pattern and overlapping cloud pattern in Ming and Qing Dynasties, the cloud pattern has experienced thousands of years and different dynasties. Its forms include abstract cloud and thunder pattern, superimposed group cloud, ethereal cloud, and repeated combination in unit form. And these different kinds of traditional moire can extract different moire design elements through concrete, abstract and reconstruction methods, and these newly extracted moire elements can break through the stereotyped thinking formed in the past, thus producing new design inspiration, so as to design more new forms of landscape sketches, so that people feel a new impact on the vision.

\subsection{Enhance cultural connotation}

From the visual depiction of cloud pattern in the primitive period to its auspicious implication, it is a kind of evolution and expression of culture, so cloud pattern is born with cultural connotation. Secondly, cloud pattern is rooted in China's five thousand years of profound cultural heritage, so it contains China's traditional civilization ideas, and these contented and auspicious civilization ideas can correctly guide people to maintain psychological balance and achieve spiritual comfort. Therefore, the application of traditional moire to the design of landscape sketch can enhance the cultural connotation of the new design landscape sketch, so as to produce cultural resonance, so as to give people spiritual comfort.

\subsection{Enhance the artistic conception of aesthetics}

Cloud pattern originated from life, which is formed in people's concept, so it will continue to develop with the continuous progress of society and people's concept. Therefore, after the evolution of the long history, cloud pattern has a traditional aesthetic concept. Therefore, cloud pattern has the aesthetic conception of "both shape and spirit". Therefore, cloud pattern not only gives people the visual enjoyment of beauty, but also gives people a kind of beautiful feeling in God. Landscape sketch contains both practical value and aesthetic value, so landscape sketch should not only have practical function, but also give people a kind of aesthetic pleasure and enjoyment. The cloud pattern can be used in the design of landscape sketches. On the one hand, it can be inspired by the formal rules of cloud pattern in the United States. Because of the characteristics of twists, downs and thickness, the cloud pattern gives people a sense of rhythm and rhythm; on the other hand, the gradual change and rotation of cloud pattern cycle produces the wonderful effect of "singing and sighing". To some extent, it expresses the aesthetic pursuit of the traditional Chinese aesthetic concept of "vivid and vivid" and emphasizes the artistic conception of "Flowing Clouds and flowing water".

\section{APPLICATION OF TRADITIONAL MOIRE IN LANDSCAPE SKETCH DESIGN}

IIn Chinese traditional patterns, cloud pattern not merely has rich modeling, but also contains unique cultural connotation, and also has unique image charm. so it can be used as the source of design elements and inspiration. Firstly, it selects the representative moire pattern and analyzes it. Secondly, it uses the formal rules of beauty to make subtraction design on the pattern (as shown in the left figure of 4-1 and 4-2 below), so as to extract the most quintessence and representative elements in the pattern. Eventually, the most valuable elements will be extracted to transform and redesign, so as to integrate with the specific landscape sketch design.

\subsection{Design and application of moire in landscape rest seat}

Landscape rest seat is a kind of landscape sketch or structure used to "sit" in the landscape environment. It has various forms, and the landscape rest seat can be flexibly arranged. It can be seen everywhere in the landscape space environment, such as flower beds, corridors and low walls. The landscape rest seat is an essential element in the landscape environment. The landscape rest seat integrates beauty and practicality. It can provide people with a rest space.

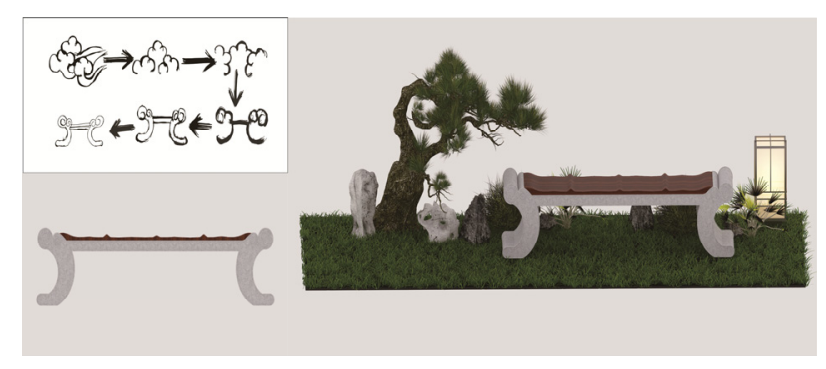

Figure 1. application of moire in landscape seat design

Because of the above importance of landscape rest seats, it is necessary to redesign the landscape rest seats. In the design of the landscape rest seat in Figure 4-1 above, we first find the most significant element of moire, and then connect this element with the structure of the landscape rest seat, so as to continuously expand and enrich the symbols of Moire elements, so as to get a new form of landscape rest seat. The landscape seat adopts the combination of stone and wood, and the main structure adopts stone, ensuring the firmness of the seat and giving people a sense of security. Secondly, the wood material is used on the seat surface, which can avoid the disadvantage of stone being cold in winter, and the use of wood on the seat surface can develop strengths and avoid weaknesses. At the same time, it can give people a sense of affinity. Cloud pattern is applied to the design of 
landscape rest seats. First of all, from the shape of the seats, its shape is simple and gives people a sense of relaxation. Second, from the form of beauty, there are not only the contrast of curves and straights, but also the contrast of materials. The most substantial thing is to complement each other, harmony and Appropriateness. Finally, because cloud pattern has a long cultural history, it is able to give people a sense of relief a different kind of charm, so as to create a variety of artistic effects.

\subsection{Design and application of moire in outdoor tea table}

Outdoor tea table is mainly placed in the outdoor environment space, which can be applied for people to place tea sets, fruit plates and other similar items when chatting at small parties. Because the outdoor tea table is placed in the outdoor environment space, so the tea table is generally placed on both sides of the seat, in addition, the tea table also presents the characteristics of square. People doing daily activities more frequently around it, so in the design of the coffee table will be its edges and corners by line smooth processing. Because the outdoor tea table not only has practical function, but also has landscaping function when placed outdoors. So the quality of tea table often directly affects the level of people's party.

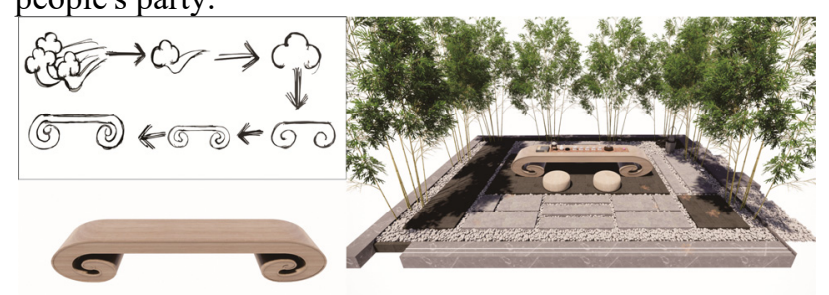

Figure 2. application of moire in outdoor tea table

A delicate and beautiful tea table can not only reflect the style of the space, but also make the finishing point. The design of the tea table in Figure 4-2 combines the moire elements with the structure of the tea table, which not only conforms to the regular characteristics of moire in structure, but also has some subtle parts and moire patterns. The moire is soft, plump, smooth, tactful and tortuous. The material of the tea table mainly made of wood, because the style belongs to the new Chinese style, so wood is more suitable for the style attribute. The tea table placed outdoors, playing a practical function, has the aesthetic characteristics of art sketches, but also can create a mood space at the same time.

\subsection{Design and application of moire in landscape lamp}

The landscape lamp is also known as decorative lighting. It mainly uses the "virtual" of light effect and the "real" of modeling to present different landscapes day and night. On the one hand, it has lighting function, on the other hand, it has decorative function. As one of the important facilities in the landscape environment space, people not only emphasize the functionality of the landscape lamp, but also put forward requirements for its artistry and culture as well as the humanization of the product itself.

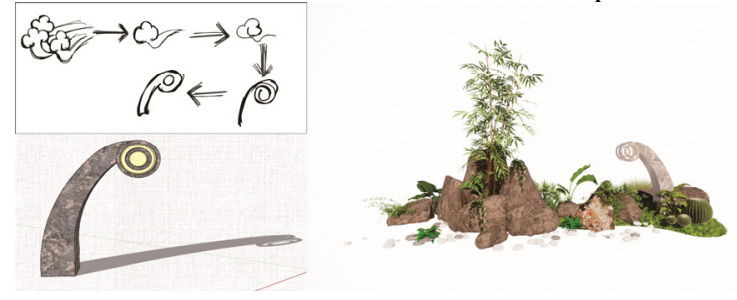

Figure 3.Application of moire in landscape lamp

As is shown in Figure 3, the landscape lamp mainly applies the moire pattern to the structure of the landscape lamp. From the perspective of view of design technique, it is a combination of Moire elements and stone. Although the volume of the landscape lamp is small, because the moire itself is rich in cultural connotation, the landscape lamp can also reflect a certain auspicious meaning, so it adds a little sentiment to the landscape environment.

\section{Conclusion}

Landscape sketch is an essential component of landscape environment, and also a punchline in landscape environment. To study the application of traditional cloud pattern in landscape sketches, we can provide more cultural background for landscape products, so as to improve the cultural connotation of landscape products; then, it can provide more design elements and inspire design inspiration, so as to make up for the single design form of landscape sketch; finally, from the perspective of application, we will take a traditional pattern as the research object to find out The essence of the design can inspire our design thinking by introducing it into the new design. Consequently, more and more excellent landscape products are devised to meet the increasing demand of people for landscape environment.

\section{REFERENCES}

1. Liu Yasai. Application of traditional Chinese patterns in landscape sketches [J]. Popular literature and art, 2017, (4): 75

2. Wu Si. Application of traditional patterns in modern urban decoration -- a case study of urban cultural landscape in Huzhou City, Zhejiang Province [J]. Business story, 2015, (8): 76-77

3. Duan Yan, Yang Xin. On the application of traditional patterns in Modern Landscape Architecture [C] / / Proceedings of 2012 annual meeting of Chinese society of landscape architecture. North China University of technology, 2012:25-29

4. Wang lulu. Research on the application of traditional Chinese patterns in urban public space landscape design [D]. Shenyang Normal University, 2015

5. Xie TIANYAO. On the symbolism of traditional Chinese patterns [J]. Shenhua, 2018, (2): 147 
6. Niu Lina. Application of traditional Chinese patterns in landscape sketches [J]. Grand View of fine arts, 2015, (7): 59

7. Zhu Xingxing. Analysis of the application of Chinese traditional auspicious plant patterns in landscape design $[\mathrm{J}]$. Modern decoration (Theory), 2015, (9): 73

8. Elena. The application of auspicious patterns of traditional Chinese decoration in modern urban landscape design $[\mathrm{J}]$. Modern decoration (Theory), 2014, (8): 64

9. Sun Lei. The origin of traditional "cloud pattern" and its artistic enlightenment [j]. Lantai world, 2015, (15): 149-150K. Elissa, "Title of paper if known," unpublished.

10. hen Gang, Wang Zixuan, Chen long. The value of practical beauty to traditional moire aesthetics [J]. Design art, 2017, (3): 101-104 